\title{
L'échec programmé de la Loi sur les Pauvres
}

The Inevitable Failure of the Poor Law

\section{Anne-Catherine de Bouvier}

\section{(2) OpenEdition}

\section{Journals}

Édition électronique

URL : http://journals.openedition.org/rfcb/215

DOI : $10.4000 /$ rfcb. 215

ISSN : 2429-4373

Éditeur

CRECIB - Centre de recherche et d'études en civilisation britannique

Édition imprimée

Date de publication : 1 septembre 2014

Pagination : 17-31

ISSN : 0248-9015

Référence électronique

Anne-Catherine de Bouvier, "L'échec programmé de la Loi sur les Pauvres », Revue Française de

Civilisation Britannique [En ligne], XIX-2 | 2014, mis en ligne le 15 février 2014, consulté le 19 avril 2019 URL : http://journals.openedition.org/rfcb/215 ; DOI : 10.4000/rfcb.215

Ce document a été généré automatiquement le 19 avril 2019

\section{c) (†) $९$}

Revue française de civilisation britannique est mis à disposition selon les termes de la licence Creative Commons Attribution - Pas d'Utilisation Commerciale - Pas de Modification 4.0 International. 


\title{
L'échec programmé de la Loi sur les Pauvres
}

\author{
The Inevitable Failure of the Poor Law
}

Anne-Catherine de Bouvier

1 Selon les propos mêmes de son principal concepteur, George Nicholls, la Loi sur les Pauvres irlandaise n'était pas destinée soulager le pays dans le cas où une famine frapperait l'Irlande dans son ensemble : elle n'en avait ni les moyens, ni la finalité.

The strict limitation of relief to the workhouse may possibly be objected to, on the ground that extreme want is found occasionally to assail large sections of the population in Ireland, who are then reduced to a state bordering upon starvation; and ought therefore, it might be asserted, be relieved at the public charge, without being subjected to the discipline of the workhouse. This, however, is an extreme case, and it would not be wise, I think, to adapt the regulation of Poor Law administration in Ireland to the possible occurrence of such a contingency. In a period of famine, the whole population become in a great degree destitute; but it surely would not be expedient to hold out an expectation, that, if such an event should unhappily occur, support for all would be unconditionally provided at the public charge. [...] The occurrence of a famine, however, if general, seems to be a contingency altogether above the powers of the Poor Law to provide for. ${ }^{1}$

2 C'est pourtant de ce cadre que le gouvernement britannique fit la norme absolue de ce que devait être le secours aux victimes de la Famine, dans l'esprit, la lettre, et l'organisation, même si la réalité de la situation imposa des écarts, tant aux administrateurs locaux, qu'aux autorités de Dublin et au gouvernement britannique. C'est ce paradoxe que cet article cherche à explorer.

Depuis l'Union de 1801, la classe politique britannique, dans sa grande majorité, percevait l'Irlande comme un élément à part au sein du Royaume-Uni, tout en cherchant à promouvoir, non pas un développement spécifique pour une île largement perçue comme arriérée à tous points de vue, mais une homogénéisation via une intégration dans les cadres légaux, économiques et sociaux de la Grande-Bretagne, voire de l'Angleterre. 


\section{Quelle « question irlandaise »?}

4 Si les années 1800-1810 furent marquées par des enjeux sécuritaires dans le contexte des guerres napoléoniennes, les années 1820 par le questionnement démocratique (qui aboutit à l'Emancipation Catholique de 1829, et à la Réforme électorale de 1832), les années 1830 furent dominées, à l'échelle du Royaume-Uni tout entier, par des préoccupations d'ordre socio-économique. La situation de l'Irlande avait déjà fait l'objet de divers commentaires de voyageurs, rapports parlementaires, propositions de lois, etc. Parmi les différents travaux, on relèvera des enquêtes importantes, qui générèrent de très volumineux rapports - et dont les titres indiquent souvent l'orientation dominante : la première étude d'envergure fut conduite en temps de paix, par le Select Committee on the State of Disease, and on the Condition of the Labouring Poor, in Ireland (1819). Cette première enquête parlementaire déclencha une guerre de pamphlets, mais ne fut suivie d'aucune traduction législative significative. Tel fut également le sort des travaux du Select Committee on the Condition of the Labouring Poor in Ireland, and on the Application of Funds for their Employment, paru en 1823, mais probablement victime de la montée en puissance de la demande d'émancipation catholique. Le très volumineux rapport du Select Committee in the State of the Poor in Ireland, and on the Means of Improving their Condition, paru en 1830, marqua l'ouverture d'une période d'intense réflexion sur la question socio-économique en Irlande, et, à partir de 1832, d'une grande activité législative. Celle-ci se caractérisa par une série de lois ad hoc, visant les dispositifs existants: on légiféra sur les différentes institutions charitables subventionnées, sur les travaux publics (auxquels le gouvernement avait ponctuellement recours pour fournir aux plus démunis un travail salarié dans des zones subissant des difficultés particulières), et sur les grands jurys, chargés de la fiscalité locale à l'échelle des comtés.

5 Ces réformes ponctuelles paraissent minimes au regard, par exemple, de l'ambitieux titre programmatique du rapport de 1830 . Cela s'explique par divers facteurs, le premier étant le coût potentiel d'une politique sociale de grande ampleur applicable à l'Irlande. En second lieu, les mesures généralement proposées², indépendamment même de leur coût, relevaient d'un interventionnisme étatique qui n'était pas dans les mœurs de l'époque. Enfin, les réflexions sur la Loi sur les Pauvres anglaise, sur laquelle nous allons revenir, imposèrent un nouveau cadre idéologique, qui freina puis orienta l'action politique dans un sens très différent du cadre interprétatif et des pistes suggérées jusqu'alors. Ainsi, le rapport commandé à George Nicholls par Lord John Russell (alors Home Secretary), brièvement évoqué plus haut, s'inscrivit-il en opposition aux travaux de la Royal Commission on the Condition of the Poorer Classes, parus en 1835, et témoignant d'une approche méticuleuse, longue, et systématique, de la question in situ.

6 Sans nous attarder au contenu et propositions de ces différentes investigations officielles, nous relèverons juste quelques points qui se dégagèrent, et formèrent en quelque sorte le prisme par lequel la classe politique britannique, dans sa majorité, percevait la « question irlandaise.» Il s'en dégageait la vision d'une société économiquement arriérée, essentiellement rurale, fondée sur une culture vivrière pratiquée massivement (la pomme de terre), peu ou pas tournée vers l'économie marchande, et dont l'industrie était chétive ou inexistante ; seul le nord-est du pays semblait faire exception. La population était donc dans un état de grande vulnérabilité, et pouvait à tout moment basculer de la pauvreté vers l'indigence. Cette population, majoritairement catholique, était ignorante, souvent 
encline à la paresse et à la rébellion. Ces caractéristiques étaient fréquemment attribuées à une défaillance des élites locales : propriétaires non résidents (absentee landlords) et indifférents à la bonne santé économique et sociale de leurs domaines, et corps intermédiaires corrompus (jobbers) détournant la fiscalité locale à des fins personnelles. La démographie irlandaise était perçue comme galopante et excessive, et ce d'autant plus que, en l'absence d'emplois alternatifs dans l'industrie, cette population croissante restait sur la terre, pour assurer sa survie grâce au lopin de pommes de terre selon certains, par entêtement politique irrationnel selon d'autres. Il en résultait un système économiquement pervers de sous-locations, qui avait créé une classe très vulnérable en bout de chaîne, les cottiers (ou cotters), dont la présence rendait impossible toute réforme des pratiques agricoles. Enfin, le pays était sillonné d'imposteurs (sturdy beggars), recevant la charité d'autres pauvres, à une échelle qui représentait une ponction énorme de la production agricole. Bien entendu, il ne s'agit là que d'un résumé schématique.

\section{Le modèle et la copie}

7 Les parlementaires, relayant par là une préoccupation croissante des contribuables anglais, étaient également fort soucieux de la condition des pauvres en Angleterre, dont témoignait l'augmentation continue du coût de la Loi sur les Pauvres anglaise. La Loi sur les Pauvres anglaise, adoptée sous le règne d'Elisabeth, avait connu bien des évolutions, dans les textes et dans la pratique. En théorie, au début des années 1830, un indigent et sa famille pouvaient être secourus dans la paroisse, aux frais des contribuables qui acquittaient les poor rates, en échange d'un travail d'intérêt public tel que l'entretien des haies et des clôtures; en pratique, il s'avérait souvent fastidieux de trouver semblables occupations à leur donner, et de nombreuses paroisses avaient fini par distribuer de l'aide, en espèces ou en nature, sans contrepartie. Il existait également des institutions closes (workhouses, asiles divers), inégalement réparties et gérées sur le territoire. Le coût de la Poor Law était partout en augmentation, et était devenu difficile à supporter dans les secteurs industriels, où une partie importante de la population pouvait être paupérisée à tout moment par un arrêt de la production ou une baisse des salaires ${ }^{3}$.

8 C'est sur la base de ce constat que travailla la Commission présidée par Edwin Chadwick, dont les propositions déterminèrent l'élaboration de l'Amendement de la Loi sur les Pauvres de 1834 (également appelé Nouvelle Loi sur les Pauvres), avec une approche spécifique de la question. Selon les auteurs de ce rapport, le secours aux indigents était devenu pervers: sachant pouvoir compter sur ce secours, les pauvres n'étaient pas encouragés à travailler, mais plutôt à rester chez eux, tandis que les employeurs pouvaient y voir un moyen de ne pas payer aux ouvriers un salaire suffisant pour assurer leur survie et celle de leur famille. Sur la base de cette vision du paupérisme, un paupérisme choisi d'un côté, alimenté (au sens propre comme au sens figuré) de l'autre, les commissaires élaborèrent le less eligibility principle, ou principe de répulsion. Solliciter l'aide publique ne devait être que le recours ultime ; il s'agissait donc de rendre le secours aussi déplaisant et contraignant que possible. Ceci devait être obtenu par la fin du outdoor relief, qui permettait aux indigents de rester chez eux, et la généralisation des workhouses ( indoor relief) : celles-ci devraient obéir aux mêmes principes et aux mêmes règles dans toute l'Angleterre. Les conditions de vie étaient strictes: séparation des familles, logement des femmes, des enfants et des hommes en dortoirs séparés, uniforme grossier, interdiction du tabac, de l'alcool et des jeux de hasard, nourriture inférieure en qualité et 
en quantité à ce que pouvait se procurer le plus mal payé des travailleurs indépendants (ce qui, dans ce contexte, signifie qu'il subsiste par son seul travail) ${ }^{4}$. Ce secours devait également être assorti d'une obligation de travail. Une discipline et un emploi du temps stricts régissaient la journée, avec quelques aménagements selon les catégories d'indigents. Afin de mettre le dispositif en œuvre, les paroisses furent regroupées en Unions, dont chacune devait contenir une workhouse. L'ensemble fut placé au niveau national sous le contrôle de Commissaires aux Pauvres, relayés localement par des administrateurs rémunérés.

9 Cette loi avait une double finalité : économique et sociale. Il s'agissait d'une part de réduire le coût de ce filet de sécurité et, partant, de libérer les sommes dépensées à ce titre à des fins productives. D'autre part, il s'agissait de mettre en œuvre une forme d'ingéniérie sociale, en incitant fortement les patrons à payer le travail à ce qu'on estimait alors être sa véritable valeur, tout en contraignant à se remettre au travail une partie, que l'on espérait et supposait importante, de la population ${ }^{5}$.

Dans la continuité de ces débats anglais, l'adoption d'une semblable Loi sur les Pauvres pour l'Irlande était de plus en plus envisagée, avec un certain succès auprès des parlementaires britanniques, dont beaucoup estimaient que la pauvreté irlandaise pesait lourd sur le contribuable anglais. En effet, de nombreux Irlandais travaillaient en Angleterre, de manière permanente, ou saisonnière, à la morte saison agricole. Certains observateurs anglais firent valoir que les Irlandais, indifférents au confort, faisaient baisser la rémunération générale du travail en acceptant des salaires moindres, ce qui avait pour effet de priver des Anglais d'emplois, et d'en rejeter d'autres sur l'aide publique. Et si, pour beaucoup, les campagnes irlandaises étaient parcourues de mendiants imposteurs feignant l'infirmité, pour certains, les campagnes anglaises étaient parcourues de mendiants irlandais qui, ayant cousu leurs gains dans l'ourlet de leur pantalon ou la doublure de leur veste, se faisant "rapatrier $»^{6}$ en Irlande aux frais du contribuable anglais 7 . Dès lors, il s'agissait de faire en sorte que l'Irlande finançât ses propres pauvres.

11 Les parlementaires irlandais étaient quant à eux nettement plus réservés sur cette proposition, pour des raisons diverses. Au premier rang de celles-ci, bien que ce ne fût pas toujours exprimé aussi clairement, intervenait la question du financement et de la fiscalité d'une telle mesure. Lors des très nombreux débats parlementaires, on entendit ainsi certains déclarer que : "Ireland is too poor for a Poor Law.» Il y eut également des objections d'ordre plus idéologique ou philosophique. Ainsi Daniel o'Connell exprima-t-il à plusieurs reprises une objection d'inspiration chrétienne et patriotique, terme que nous employons ici de préférence à nationaliste. Selon lui, la charité qui dominait la vie sociale des classes les plus pauvres en Irlande était la preuve d'une profonde fidélité aux valeurs chrétiennes, fidélité pas encore pervertie par des valeurs mercantiles et individualistes, et sur laquelle la loi humaine ne devait pas tenter d'intervenir.

L'adoption et la mise en œuvre de la nouvelle Loi sur les Pauvres anglaise fut une période de latence pour la question irlandaise, non dans les réflexions (il y eut de nombreux pamphlets, et des propositions de loi), mais dans la décision. Le gouvernement reprit l'initiative, ayant acquis la conviction que la grande pauvreté irlandaise était soluble dans le modèle anglais, si récent fût-il. C'est ainsi qu'en 1836, George Nicholls, l'un des commissaires de la Loi sur les Pauvres, fut dépêché en Irlande par Lord John Russell, avec pour mission explicite d'y étudier la transposabilité du dispositif récemment adopté en Angleterre - balayant ainsi les volumes d'enquête sur ce sujet. Fidèle à l'esprit et à la 
lettre de sa mission, au terme d'une visite éclair de six semaines, Nicholls conclut de manière affirmative.

13 Le rapport de Nicholls témoigne de l'ambiguïté initiale au cœur du dispositif envisagé puis adopté pour l'Irlande, ainsi que d'une vision idéalisée des vertus du nouveau système anglais. Sa perception des masses irlandaises reprend et condense les lieux communs partagés alors par une grande partie de l'opinion publique britannique : «They seem to feel no pride, no emulation, to be heedless of the present, and reckless of the future. They do not [...] strive to improve their appearance, or add to their comfort ${ }^{8}$. $)$ On sent ici poindre le trope du sauvage, voire de l'animalité, que Nicholls poursuit un peu plus avant dans sa description :

On entering a cottage, the woman and children are often seated on the floor, surrounded by pigs and poultry, in the midst of filth - the man lounging at the door, to approach which it is necessary to wade through mud; yet, he is too indolent to make a dry approach to his dwelling, although there are materials fit for the purpose close at hand; his wife is too slatternly to sweep the place in which they live, or remove the dirt and offal, however offensive, from the floor. ${ }^{9}$

Le paysan irlandais, tel que décrit par Nicholls, est un être bestial, inconséquent, existant dans une sorte d'hébétude animale, vivant de la terre mais sans application, tout prêt qu'il est à laisser la récolte pourrir sur pied pour se précipiter à une foire ou à un mariage : "they hurry off in search of the excitement and the whiskey which abound on such occasions..$^{10}$ " Paresse, alcoolisme, inconséquence, telles sont selon Nicholls les causes d'une économie peu productive, et de la vulnérabilité chronique d'une grande partie de la population, avec ses corollaires : l'imposture, la violence, la rébellion.

mède qu'il préconise pour rompre ce cercle vicieux est, sans surprise, l'adoption d'une Loi sur les Pauvres calquée sur le modèle anglais, à quelques différences près ${ }^{11}$. Selon lui, cette mesure serait de nature à remettre les paysans au travail et à responsabiliser les propriétaires, créant ainsi un cercle vertueux, au terme duquel l'agriculture deviendrait productive, ce qui libèrerait des bras et des capitaux pour des investissements et des projets industriels. Une telle loi créerait ainsi l'impulsion nécessaire au changement, tout en servant de mesure d'accompagnement de la société irlandaise dans la période de transition. Il est ici davantage question d'ingéniérie sociale et politique : éliminer à terme le paupérisme, en en traitant les causes : «A Poor Law seems also to be necessary, as a first step, towards effecting an improvement in the character, habits and social conditions of the people ${ }^{12}$. "

16 Le propos de Nicholls glisse fréquemment de la dimension socio-économique à la dimension nationale ${ }^{13}$, et compare fréquemment les pratiques anglaises et irlandaises, où l'Irlande figure tantôt comme une version archaïque de l'Angleterre (celle d'avant la Loi sur les Pauvres d'Elizabeth, ou celle d'avant la réforme de 1834), tantôt comme une contrée exotique, peuplée de sauvages susceptibles d'être éduqués et civilisés. En réponse à une objection - concentrer des Irlandais en un même espace clos risquerait de générer de la violence - Nicholls affirme : " the Irish are easily governed, and easily led; and as in the workhouse they would be free from the influence of spirits, and other excitements, I anticipate no difficulty in establishing an efficient system of discipline and classification. ${ }^{14} »$ Il faut ici préciser que, dans une logique où la workhouse serait le seul mode de secours aux indigents, il en est proposé une classification, des infirmes et jeunes enfants aux mendiants robustes, à laquelle s'attachent différents degrés de discipline et différentes quantités de travail exigé. 
17 Nicholls semble sincèrement convaincu des vertus civilisatrices du projet de Loi sur les Pauvres. Ainsi, il recommandait que, dans les zones les moins peuplées, la workhouse fasse physiquement partie d'un réseau institutionnel visible: elle devrait être érigée à proximité du poste de police principal afin de faire bénéficier ceux qui l'approcheraient d'un modèle d'ordre, comporter ou être située à proximité d'un dispensaire et d'une école, et inclure un potager modèle. Il s'agissait donc tout à la fois de réprimer, policer, réformer, (ré)éduquer, par les faits et par l'exemple. "The Union established would thus become some kind of colony, a kind of centre of civilization, and the Unions collectively might be made important engines for effecting improvement in the conditions and habits of the Irish people. ${ }^{15}$ » On pourrait faire crédit à Nicholls d'avoir sérieusement envisagé des modèles alternatifs, tels que les colonies de pauvres de Belgique ou des Pays-Bas. Toutefois, outre le traitement qu'il accorde à ces modèles (voir note $n^{\circ} 1$ ), on peut difficilement éviter le poids des termes "colonie » et "civilisation" dans le contexte des relations angloirlandaises. La question du paupérisme semble secondaire ou ancillaire dans ce type de propos ; les indigents seraient ici transformés en fer de lance d'une révolution culturelle visant à une assimilation des deux pays par la reconquête sociale et idéologique de l'Irlande.

Ce rapport constitua la base technique et idéologique de la Loi sur les Pauvres qui fut adoptée pour l'Irlande en 1838, et dont on peut résumer ainsi les principales dispositions ${ }^{16}$. Tout d'abord, tout le dispositif de secours aux indigents fut, dès le préambule, placé sous l'autorité et le contrôle constant des Commissaires nommés en vertu de la loi anglaise de 1834 ; ceux-ci avaient la possibilité de nommer des Commissaires adjoints ( Assistant Commissionners), placés sous l'autorité d'un des commissaires, résidant en Irlande; lequel était investi de tout pouvoir d'application de la loi, hormis en ce qui concerne les règles en vigueur dans les workhouses, lesquelles devaient être validées par le Commissariat aux Pauvres (Board of Commissioners) dans son ensemble; ce commissaire était sous l'autorité directe d'un département $\mathrm{d}^{\prime} \mathrm{Etat}^{17}$. On voit bien la dimension assimilationniste et centralisatrice à l'oeuvre dans la loi. De surcroît, toutes les institutions existantes antérieurement, dès lors qu'elles étaient en tout ou en partie financées par des deniers publics, furent également placées sous l'autorité du Commissariat ${ }^{18}$. La loi prévoyait la création d'Unions, subdivisées en districts électoraux pour l'élection des gardiens localement responsables de la mise en œuvre et de la gestion du dispositif, et devant chacune être dotée d'une workhouse, qu'il s'agisse d'une construction spécifique, ou de la conversion d'un bâtiment à cet usage; à terme, celle-ci devait se substituer à toute autre institution, dans la logique de la loi anglaise.

Les poor rates assuraient le financement: celles-ci étaient calculées, votées et collectées dans chaque district électoral, en fonction des besoins de l'Union, et au prorata de la valeur annuelle de la terre ou des locaux; les rates étaient acquittés par l'occupant (si celle-ci était inférieure à $€ 5$, les rates étaient acquittées par le propriétaire), et déductibles pour moitié du loyer ou du fermage jusqu'à concurrence d'une livre ${ }^{19}$. Cette disposition répondait à une double préoccupation : ne pas trop pénaliser les contribuables irlandais, tout en contraignant les propriétaires à s'acquitter mieux, ou davantage, de leur mission économique et sociale locale. La Loi sur les Pauvres irlandaise incitait ainsi les propriétaires à "consolider" les domaines (euphémisme par lequel on désignait l'éviction des métayers ou occupants surnuméraires), tout en leur donnant les moyens, du moins en théorie, de le faire en limitant les risques de réactions violentes de la part de la population concernée, en offrant à cette dernière un filet de sécurité. Enfin, la loi 
établissait toute une bureaucratie salariée en charge de l'administration du dispositif tant au niveau de l'exécution que du contrôle, plaçant ainsi sous coupe réglée les élites locales irlandaises, en même temps que les indigents. On peut ici avancer que cette loi répondait au moins autant à un objectif de réforme agraire, voire à un objectif socio-politique à moyen terme, qu'à la nécessité de pallier l'indigence.

Cette loi fut adoptée à l'initiative de Lord John Russell, alors Home Secretary, et qui fut Premier ministre dès le printemps 1846, abordant la Famine avec ce bagage administratif et intellectuel. Elle reflète à la fois l'idéologie whig, l'influence des théories utilitaristes ${ }^{20}$, et la confiance (on serait presque tenté de parler de foi) en la suprématie du modèle anglais, dont témoignait a contrario l'indigence irlandaise elle-même. En d'autres termes, il existait bel et bien des différences profondes entre les deux pays, mais si le mal ne provenait pas toujours, ou du moins pas systématiquement des mêmes causes, il était toutefois susceptible d'être éradiqué par les mêmes remèdes, ceux-ci étant posés comme assimilationnistes, dans la logique de l'Union. L'essentialisme cohabitait sans peine avec la rationalité arithmétique, prescriptive et punitive du dispositif anglais, lequel était perçu et posé comme un modèle universel. La seule réserve de Nicholls portait, précisément, sur la survenue d'une famine à l'échelle de l'île tout entière.

Il n'y avait pas de proposition alternative : l'hypothèse d'un désastre de ce type n'avait pas été incluse dans la mission de Nicholls, et ne semble pas avoir été concrètement envisagée dans les cercles gouvernementaux, malgré la prévalence des propos alarmistes sur l'état économique et social de l'Irlande, ou peut-être à cause même de ceux-ci. En effet, et indépendamment de l'existence d'un sentiment anti-irlandais, il faut ici avancer la possibilité que la classe politique et l'opinion publique britannique s'étaient habituées aux descriptions d'une précarité ou d'une misère extrême aux tropes désormais récurrents : " miserable hovels, unfit to shelter our swine », « the most extreme distress » (ainsi que "surplus population»), avaient émaillé les divers rapports et pamphlets. On parlerait alors de " outrage fatigue ». Il faut ajouter cette hypothèse à la réelle méfiance vis-à-vis des propriétaires irlandais et de leurs relais administratifs et sociaux, ainsi qu'une méconnaissance des classes populaires (qu'elles soient ouvrières ou paysannes) de la part des classes dirigeantes, pour comprendre l'ampleur du gouffre entre les informations reçues et les mesures adoptées ou écartées. Tout cela vint s'ajouter au substrat idéologique de l'époque, teinté de malthusianisme, d'utilitarisme, et de laisser-faire économique.

\section{L'esprit, la lettre, et la réalité}

L'ampleur du désastre contraignit le gouvernement à adopter des mesures déviant des principes et de la lettre de la Loi sur les Pauvres irlandaise, ces déviances étant a priori posées comme ponctuelles, exceptionnelles et, partant, limitées dans le temps. Sir Robert Peel, Premier ministre tory au début de la Famine, en lien avec le Château de Dublin, envisagea la possibilité de recourir à des travaux publics, et ce dès novembre 1845. La politique de travaux publics n'était pas chose nouvelle en Irlande, et dès 1831 le Board of Works avait été créé, regroupant les différents départements qui auparavant supervisaient ces travaux. Pour Peel et le Lord Lieutenant, le but était de soulager la population en lui permettant de trouver un emploi rémunéré (qui lui permettrait d'acheter de quoi se nourrir, en compensation de la perte de la culture vivrière), tout en réalisant des infrastructures de nature à promouvoir un développement à plus long terme du pays. 
C'était en quelque sorte une réponse classique à une crise ponctuelle, la différence étant son étendue géographique. En mars 1846, le Parlement confirma le rôle du Board of Works dans l'organisation des mesures d'aide par les travaux publics. Certains travaux devaient être financés au niveau des baronnies concernées, par la fiscalité locale; les routes, en revanche, étaient financées localement pour moitié, le reste l'étant par le Trésor (halfgrant system). Dans les deux cas, ce dernier avançait les fonds, après approbation du projet par le Lord Lieutenant. Ces mesures devaient cesser à l'été 1846 ; d'une part, on n'anticipait pas un retour du mildiou, et d'autre part, le gouvernement whig qui accéda au pouvoir suite à la chute de Peel était davantage sensible aux récriminations du Trésor qu'aux rapports venant d'Irlande.

Face à l'ampleur du désastre, la politique de travaux publics fur reconduite en août 1846, mais assortie de davantage de contraintes et de contrôles, sous l'impulsion de Charles Trevelyan $^{21}$. Le Labour Rate Act mit un terme à tout financement par le Trésor, qui se contenterait désormais d'avancer les sommes aux baronnies, lesquelles le rembourseraient; il plaça également le contrôle des chantiers et des employés sous l'autorité totale du Board of Works, doté de personnel salarié en conséquence ${ }^{22}$. De plus, cette loi remplaça le salaire journalier par un paiement à la tâche ; cette disposition fut particulièrement impopulaire, d'autant que les prix alimentaires s'envolaient, réduisant ainsi la quantité de nourriture que les ouvriers pouvaient se procurer. Ceci eut pour effet de les affaiblir et, partant, de diminuer leurs capacités physiques et leur rémunération, alors que l'hiver 1846-1847 fut particulièrement rude, au point que des chantiers durent s'arrêter plusieurs jours, causant ainsi une perte supplémentaire de salaire. Il en résulta une nette augmentation de la mortalité.

Aussi, dès le mois de mars 1847, le gouvernement annonça-t-il une réduction des travaux publics, et imposa-t-il une réduction programmée du nombre d'ouvriers employés en vue d'une cessation totale de ce programme au $1^{\mathrm{er}}$ mai. Le secours devait prendre la forme d'aide alimentaire, distribuée par des soupes populaires (soup kitchens) ${ }^{23}$. Cette fois encore, la responsabilité financière du programme devait rester locale, avec quelques discrètes exceptions pour venir en aide aux secteurs les plus touchés et les plus appauvris de l'ouest de l'Irlande. Ces soupes populaires tardèrent à se mettre en place, et en mai, à peine les deux tiers de celles qui avaient été prévues (pour mars) étaient ouvertes. L'arrêt du dispositif était prévu pour août 1847, dans l'anticipation d'une bonne récolte, et en prévision d'un transfert total du secours vers la Loi sur les Pauvres. De fait, l'organisation administrative de ces soupes populaires avait été calquée sur le dispositif de la Loi sur les Pauvres, respectant les limites des Unions et des districts électoraux. Dans les endroits les plus pauvres, elles continuèrent d'opérer jusqu'à la fin du mois de septembre, cette aide étant réservée aux invalides. L'aide médicale devait également être prise en charge par la Loi sur les Pauvres, malgré l'arrivée de l'hiver, et la recrudescence et la virulence de maladies telles que le typhus, la dysenterie et le scorbut, largement provoquées et aggravées par la malnutrition ou la sous-nutrition.

La Loi sur les Pauvres, en effet, était le seul cadre que le gouvernement admettait, craignant que les Irlandais ne considérassent le secours comme un droit acquis, aux dépens du Trésor britannique. C'était également un rempart contre l'imposture, du moins potentielle - celle des pauvres, et celle des propriétaires. Il faut ici se souvenir de l'arrière-plan idéologique des deux lois (1834 et 1838) pour comprendre la suspicion avec laquelle les nouvelles d'Irlande étaient généralement reçues à Londres, et se souvenir de l'image du paysan irlandais paresseux et imprévoyant, doublée de celle du propriéraire 
vénal et négligent, pour comprendre que le lieutenant-colonel Jones, alors président du Board of Works, ait écrit à Trevelyan que " farmer, priest, landlord and tenant all make strong attempts to squeeze something out of the government's purse ${ }^{24}$. » La certitude que tout système de secours à caractère automatique encourageait l'indolence, et que toute prise en charge de la crise irlandaise par le Trésor empêcherait l'île d'y faire face (et on pensait qu'elle le pouvait), et de se réformer, peut expliquer en partie cette obstination a priori surprenante : se fonder sur une loi dont la conception avait exclu cette hypothèse.

Le dispositif de la Loi sur les Pauvres, d'ailleurs, fut mis à mal par les événements. Si, dans les premiers temps de la Famine, les workhouses demeurèrent quasiment vides, elles ne tardèrent pas à se remplir. A partir de l'hiver 1846-1847, elles étaient saturées et, localement, nombre de gardiens firent ce qu'ils purent pour soulager ceux qui le se présentaient - y compris par la distribution de nourriture (et parfois sur leurs fonds propres) quand les installations ne pouvaient plus accueillir personne, et ce en dépit même de l'interdiction de toute forme de outdoor relief.

Cette restriction fut partiellement levée en juin 1847, par un amendement à la Loi sur les Pauvres, qui autorisa des dérogations, pour une période maximale de deux mois, permettant que les personnes incapables de travailler (personnes âgées, infirmes, veuves ayant au moins deux enfants légitimes à charge) pussent recevoir une aide alimentaire en dehors de la workhouse; cette aide était toutefois purement alimentaire, et excluait totalement d'autres nécessités telles que vêtements ou combustible. Les gardiens reçurent pour consigne d'acquérir des bâtiments distincts pour cette catégorie de pauvres - le financement devant toujours être assuré par les poor rates ${ }^{25}$.

A l'été 1847, les Commissaires aux pauvres recommandèrent que l'on expulsât des workhouses les infirmes, les jeunes enfants, et les personnes âgées, afin de les réserver à ceux pour lesquelles elles avaient été conçues: les indigents valides. Cette recommandation avait le mérite de la cohérence, mais elle méconnaissait la réalité des résidents. Ainsi que le note Christine Kinealy,

A condition of entry to the workhouses was that the applicants had to be destitute, that is, without either possessions or property. [...] As the winter progressed and the demand for relief increased, the policy of forcibly emptying the workhouses of certain categories of inmates to create room for able-bodied applicants continued to pose problems. Throughout the country, there were reports that many of the old, young and infirm paupers were reluctant to vacate the workhouses, some pointedly refusing to do so. The most common reason given was that they and their families and friends no longer possessed either homes, lodgings, farms, bedding or clothing. 26

29 Il faut ajouter que les workhouses devaient enterrer leurs morts dans un cercueil; en des temps de crise extrême, la possibilité d'être enterré dans un cercueil renforça l'attractivité paradoxale des workhouses. La mesure la plus impopulaire de cette loi est connue sous le nom de "quarter acre clause », ou "Gregory clause », qui rendit inéligible à toute forme de secours quiconque occupait plus d'un quart d'acre ${ }^{27}$, ainsi que sa famille. En l'absence de récolte suffisante à l'été 1847 , les cottiers qui avaient survécu jusqu'alors se virent souvent contraints d'abandonner leur lopin, avec la certitude de ne plus avoir nulle part où retourner plus tard.

30 De manière générale, dès l'été 1847, la Loi sur les Pauvres irlandaise connut un nombre de réformes, d'exceptions et d'adjonctions dont l'empilement démontre à lui seul l'inadéquation du cadre, et ce avant même qu'il n'ait commencé à prendre en charge le secours. Le Poor Law Extension Act sépara le Poor Law Board d'Irlande du Board anglais, ce en 
quoi l'on peut lire une reconnaissance d'une réalité spécifique irlandaise, autant que l'échec de la visée assimilationniste de la loi de 1838. Au début de 1848, les vingt-deux Unions dont la situation financière était la plus critique furent autorisées à recevoir une aide exceptionnelle, mais rapidement, beaucoup d'autres Unions rencontrèrent des difficultés majeures. Durant l'été 1848, les relations entre les Commissaires aux Pauvres irlandais et le Trésor britannique se tendirent. Au printemps 1849, les Commissaires ne disposaient plus de fonds; les "aides exceptionnelles" arrivaient tardivement, bureaucratiquement, et parcimonieusement, de même que les prêts et avances sur les poor rates. Afin de garantir la continuité du financement de la Loi sur les Pauvres sans solliciter davantage le contribuable anglais, le gouvernement fit adopter le Rate-in-Aid Bill (approuvé par le Parlement le 24 mai 1849). Cette loi instaurait une taxe exceptionnelle sur tous les biens assujettis à la fiscalité, à un taux homogène dans toute l'Irlande : les Unions les moins touchées financeraient désormais celles qui étaient en difficulté. Après et malgré la bonne récolte de 1849 , de nombreuses Unions restèrent cependant dans une situation financière très précaire, ayant à rembourser les avances reçues pour les soupes populaires, la construction ou l'acquisition de locaux supplémentaires, et celles reçues au titre du Rate-in-Aid ${ }^{28}$.

\section{Conclusion}

31 La Loi sur les Pauvres irlandaise n'avait pas permis de faire face à la Famine. Si les workhouses ont peu ou prou sauvé leurs occupants de la mort de faim (du moins, ceux qui n'y arrivaient pas à un stade de dénutrition trop avancé), elles ont aussi constitué des foyers de contagion. Les gardiens, par compassion ou par crainte des conséquences pénales s'ils refusaient l'asile à un indigent en danger de mort, l'amendement de 1847 ayant introduit l'obligation de secours, allèrent parfois jusqu'à accueillir près de trois ou quatre fois le nombre de personnes possible, les indigents dormant alors à plusieurs par lit, d'où une mortalité élevée ${ }^{29}$. L'obligation de travailler au sein des workhouses, ainsi que le régime disciplinaire et punitif que l'on s'attacha à y faire maintenir, qui ajoutait l'humiliation à la faim et à la maladie, ne furent pas de nature à en faire des centres de colonies modèles et exemplaires comme souhaité par Nicholls.

La loi de 1838 avait exempté du paiement des poor rates les occupants de lieux d'une valeur fiscale inférieure à $€ 5$. Certains propriétaires saisirent cette occasion pour expulser les occupants des plus petites parcelles et faire détruire leur habitation, afin d'échapper à l'impôt. D'autres y furent contraints par leur situation financière, les loyers et fermages rentrant peu et mal en période de famine. La "quarter acre clause» amplifia ce phénomène : une fois le cottier admis à la workhouse, il suffisait de détruire le logement.

Rien de toute cela n'était de nature à inspirer une quelconque gratitude à la population irlandaise dans son ensemble, comme en atteste le succès des candidats en faveur de l'abrogation de l'Union (Repeal) lors des élections législatives de 1847 et, dans une moindre mesure, le soulèvement manqué de $1848{ }^{30}$. La cotte fut fréquemment retaillée, dans un effort assez vain pour concilier les principes fondateurs de la loi, la réalité socioéconomique irlandaise, et les circonstances exceptionnelles que connut le pays pendant la Famine. L'ambiguïté fondamentale au cœur de la loi de 1838 demeura entière, et inopérante: recourir à un paradigme posé comme universel, afin de résoudre un problème complexe perçu comme exogène, en dépit de l'opposition farouche des parlementaires représentant le territoire concerné, à des fins d'acculturation. 


\section{BIBLIOGRAPHIE}

BENTHAM, Jeremy, in Michael QUINN (ed.). Writings on the Poor Laws, vol. I. Oxford : Clarendon Press, 2001.

BRUNON-ERNST, Anne. Le Panoptique des pauvres : Jeremy Bentham et la réforme de l'assistance en Angleterre. Paris : Presses de la Sorbonne Nouvelle, 2007.

CROWLEY, John, William J. SMYTH, et Mike MURPHY (eds.). Atlas of the Great Irish Famine. Cork: Cork University Press, 2012.

DUDLEY EDAWRDS, Robert et Thomas Desmond WILLIAMS (eds.). The Great Famine: Studies in Irish History 1845-52 [1956]. Dublin : The Lilliput Press, 1994.

FOUCAULT, Michel. Surveiller et punir : naissance de la prison. Paris : Gallimard, 1975.

GRAY, Peter. Famine, Land and Politics: British Government and Irish Society 1843-1850. Dublin : Irish Academic Press, 1999.

---. The Irish Famine. Londres : Thames \& Hudson, 1995.

The Making of the Irish Poor Law, 1815-1843. Manchester : Manchester University Press, 2009.

HAINES, Robin. Charles Trevelyan and the Great Irish Famine. Dublin : Four Courts Press, 2004.

KINEALY, Christine. This Great Calamity: The Irish Famine 1845-52. Boulder, CO : Roberts Rinehart Publishers, 1995 (Dublin : Gill \& Macmillan, 1994).

---. Repeal and Revolution: 1848 in Ireland. Manchester : Manchester University Press, 2009.

NICHOLLS, Sir George. A History of the Irish Poor Law, in Connexion with the Condition of the People. Londres : John Murray, 1856.

Ó GRÁDA, Cormac. Black ' 47 and Beyond: the Great Irish Famine in History, Economy and Memory. Princeton : Princeton University Press, 1998.

SIMMEL, Georg. Les Pauvres (1908). Paris : Presses Universitaires de France (Quadrige), 1998.

\section{NOTES}

1. George NICHOLLS, Three Reports, by George Nicholls, Esq., to Her Majesty's Principal Secretary of State for the Home Department, Londres: Her Majesty's Stationery Office, 1838, First Report, p. 37. Le premier rapport, daté de novembre 1836, constitue l'approche générale de la question, fondée sur les recommandations ministérielles, la propre expérience de Nicholls en tant que commissaire aux Pauvres en Angleterre, et son séjour de six semaines en Irlande. Le deuxième (daté de novembre 1837) est un supplément, qui inclut l'Ulster (province absente du premier rapport), ainsi que des réponses aux objections soulevées par son premier rapport. Le troisième (daté de mai 1838) contient des développements sur la Hollande et la Belgique, souvent citées comme des exemples alternatifs, en raison de la pratique de colonies de pauvres, où les mendiants arrêtés étaient placés de force, et où certains pouvaient, par leur travail au sein de la colonie (travail obligatoire pour tous), se constituer un petit pécule en vue de leur sortie. Toutefois, Nicholls n'en retint (sans les avoir visitées d'ailleurs, par manque de temps et en 
raison de conditions météorologiques peu favorables) que le régime disciplinaire, qu'il compare à celui des workhouses. Il fit également l'éloge des workhouses «classiques» de Belgique et de Hollande.

2. Assèchement des marécages, mise en valeur de terres non exploitées, développement des pêcheries, encouragement de l'artisanat domestique, aide massive à l'émigration vers des colonies britanniques, politique de grands travaux.

3. Il y était aussi malaisé d'y trouver que donner à faire aux indigents, qui représentât un intérêt collectif, sans concurrencer une activité commerciale, cette double notion étant au cœur du dispositif.

4. Cet extrait du rapport fait ressortir différents éléments : l'obligation absolue du travail, la vision mécaniste d'une société fonctionnant par vases communiquants, l'idée que la condition d'assisté relève souvent d'un choix délibéré, l'aspect répulsif que doit revêtir la seule alternative au travail, et enfin, une classification métonymique (et morale) des individus des classes inférieures - avec, au bas de l'échelle, le prisonnier ; en haut, le travailleur pauvre, tandis que les assistés constituent un échelon intermédiaire entre criminels et délinquants, et pauvres actifs : « The first and most essential of all conditions, a principle which we find universally admitted, even by those whose practice is at variance with it, is, that his situation on the whole shall not be made really or apparently so eligible as the situation of the independent labourer of the lowest class. Throughout the evidence it is shown, that in proportion as the condition of any pauper class is elevated above the condition of independent labourers, the condition of the independent class is depressed; their industry is impaired, their employment becomes unsteady, and its remuneration in wages is diminished. Such persons, therefore, are under the strongest inducements to quit the less eligible class of labourers and enter the more eligible class of paupers. The converse is the effect when the pauper class is placed in its proper position, below the condition of the independent labourer. Every penny bestowed, that tends to render the condition of the pauper more eligible than that of the independent labourer, is a bounty on indolence and vice. We have found, that as the poor's-rates are at present administered, they operate as bounties of this description, to the amount of several millions annually.

"The standard, therefore, to which reference must be made in fixing the condition of those who are to be maintained by the public, is the condition of those who are maintained by their own exertions. But the evidence shows how loosely and imperfectly the situation of the independent labourer has been inquired into, and how little is really known of it by those who award or distribute relief. It shows also that so little has their situation been made a standard for the supply of commodities, that the diet of the workhouse almost always exceeds that of the cottage, and the diet of the gaol is generally more profuse than even that of the workhouse. It shows also, that this standard has been so little referred to in the exaction of labour, that commonly the work required from the pauper is inferior to that performed by the labourers and servants of those who have prescribed it: So much and so generally inferior as to create a prevalent notion among the agricultural paupers that they have a right to be exempted from the amount of work which is performed and indeed sought for by the independent labourer. " Report form His Majesty's Commissioners for Inquiring into the Administration and Practical Operation of the Poor Laws, Londres : His Majesty's Sationery Office, 1834, p. 228.

5. Elle reposait également sur une approche pénale du paupérisme : le régime alimentaire et disciplinaire de la workhouse devait être à mi-chemin entre celui de la prison et celui du plus pauvre travailleur subvenant à ses propres besoins (voir note précédente). Le peuple ne s'y trompa pas, qui qualifia très vite ces établissements de Poor Law bastilles.

6. «Rapatrier » figure ici entre guillemets, puisqu'il s'agit d'un mouvement au sein d'une même entité politique, le Royaume-Uni.

7. La Loi sur les Pauvres anglaise comportait une clause de résidence (settlement), qui imposait que l'indigent soit secouru dans sa paroisse de résidence habituelle. S'il s'en trouvait éloigné, il 
devait y être reconduit, son voyage étant payé par les poor rates. Il existait des cas de saisonniers irlandais qui rejoignaient ainsi l'Irlande, même si le phénomène est bien entendu difficile à quantifier. S'il existe des travaux parlementaires qui chiffrent le coût du retour (voir, par exemple, Return of Number of Irish Poor shipped from Bristol to Ireland, 1823-31, Sessional Papers of the House of Commons, Accounts and Papers, 1831-32, ou Return of Number of Irish Poor shipped from Liverpool to Ireland, 1823-32, Sessional Papers of the House of Commons, Accounts and Papers, 1833), l'état de nécessité réelle demeure difficile à évaluer.

8. NICHOLLS, First Report, op. cit., p. 9.

9. Ibid., pp. 9-10.

10. Ibid., pp 10-11. Le thème de l'Irlandais buveur était déjà devenu un lieu commun de l'époque. Cet élément revient à de nombreuses reprises dans les analyses de Nicholls sur les causes de la misère et du sous-développement irlandais. Nicholls alla même jusqu'à voir dans la pomme de terre un aliment de nature à accroître l'envie d'alcool, ainsi que ses effets.

11. La première, et non des moindres, était que la loi irlandaise ne comportait aucune notion de droit au secours, dont on craignait qu'elle n'encourageât l'indolence (des paysans comme des propriétaires), et ne retardât les réformes agraires perçues comme nécessaires. L'autre différence majeure était que la possibilité d'être secouru n'était pas liée à une obligation de résidence (settlement), de sorte que n'importe quel indigent pouvait demander à être admis à la workhouse de l'Union de son choix. L'argument mis en avant était que l'on devait permettre à chacun d'aller chercher du travail là où il $\mathrm{y}$ en avait, sans entraver sa mobilité ; on se demande alors pourquoi semblable amendement n'avait pas été introduit dans le Poor Law Amendment Act de 1834, d'autant plus que les deux mesures relèvent d'une même approche, et d'une même cohérence. On peut ici avancer différentes hypothèses. La première serait la volonté de ne pas alourdir davantage le système ; elle est peu pertinente, compte tenu du poids de la bureaucratie créée par la Loi sur les Pauvres irlandaise, et des grandes capacités d'investigations qui lui étaient conférées. La deuxième reposerait sur l'ampleur du vagabondage : la loi s'interdirait alors à ellemême d'atteindre son cœur de cible, et les mendiants pourraient continuer d'errer faute de domicile connu, ce qui n'est pas sans vraisemblance. En troisième lieu, on peut avancer la difficulté d'identifier clairement la population, difficulté qui avait entaché les recensements de 1821 et 1831. On peut également avancer l'hypothèse d'une mise en concurrence implicite, ou d'une mise en surveillance, du travail des autorités locales chargées de l'application de la loi. Ainsi, selon Nicholls, un afflux soudain de pauvres dans la workhouse d'une Union alerterait immédiatement les autorités centrales, et témoignerait d'un laxisme évident dans l'application des règles et dans la gestion de l'établissement. Ce serait en totale cohérence avec la défiance de la classe politique britannique vis-à-vis des élites locales irlandaises - sans exclure les deux interprétations précédentes.

12. NICHOLLS, First Report, op. cit., p. 20.

13. "Nationale » est ici préférée à "régionale », adjectif qui serait plus adéquat d'un point de vue strictement juridique, mais totalement faux du point de vue de l'histoire des mentalités.

14. NICHOLLS, First Report, op. cit., p. 20.

15. Ibid., p. 31. Il s'agit également des secteurs les plus pauvres, où les paysans sont les plus précaires. L'auteur semble ignorer totalement les sentiments de rejet et de haine qu'inspirèrent très vites les workhouses à la population anglaise, haine qui fut très vite partagée par la population irlandaise (laquelle ne regardait pas la police d'un œil plus favorable), et interdisait donc à ces institutions cette vertu exemplaire et civilisatrice.

16. An Act for the more effectual Relief of the Destitute Poor in Ireland, communément appelé Poor Law (Ireland) Act, $1 \& 2$ Vic., c. 56 ; 31 juillet 1838. Le titre intégral indique le soin qui fut pris à poser la différence entre la destitution et la pauvreté.

17. Ibid. Voir articles IX, XI et XII pour les principales prérogatives des Commissaires adjoints ; voir article CXXII pour le statut du Commissaire résidant en Irlande. La loi place le dispositif sous 
l'autorité d'un "Secretary of State ", sans spécifier lequel; il est particulièrement révélateur que cette responsabilité ait incombé à l'Echiquier.

18. Il existait en Irlande une nébuleuse d'institutions (dispensaires, hôpitaux des enfants trouvés, asiles d'aliénés, houses of industry, etc.) ; certaines ne dépendaient que de fonds publics, d'autres recevaient une dotation parlementaire, généralement égale au montant des contributions privées.

19. Ibid. Voir les articles LXIII et LXIV pour la définition des biens assujettis, LXI et LXXIII pour la collecte, LXXI et LXXII pour les contribuables, et LXXIV pour la déduction.

20. Voir Jeremy BENTHAM, in Michael QUINN (ed.), Writings on the Poor Laws, vol. I, Oxford: Clarendon Press, 2001.

21. Sir Charles Trevelyan occupa le poste d'Assistant Secretary au Trésor de 1840 à 1859 ministère qu'il connaissait déjà fort bien au moment de la Famine, où son action était appréciée, comme en atteste la durée de son exercice, et dont il défendit les intérêts très fermement lors de la crise irlandaise.

22. Voir Christine KINEALY, This Great Calamity: The Irish Famine 1845-52, Boulder, CO : Roberts Rinehart Publishers, 1995 (Dublin : Gill \& Macmillan, 1994), p. 90.

23. Temporary Relief Act, février 1947.

24. Jones to Trevelyan, 2 Mai 1846, cité par KINEALY, This Great Calamity, op. cit., p. 56.

25. Voir ibid., pp. 180-181.

26. Ibid., p. 195.

27. Un quart d'acre correspond à une surface d'environ mille mètres carrés, soit une toute petite parcelle, à peine suffisante pour la culture vivrière nécessaire à une famille ne disposant pas d'autres moyens de se procurer de quoi vivre. Il faut aussi préciser qu'à chaque parcelle correspond généralement un droit de prélever de la tourbe (généralement dans une zone marécageuse plus ou moins éloignée), c'est-à-dire le combustible nécessaire au chauffage et à la cuisson des aliments.

28. En 1850 (et après un deuxième Rate-in-Aid pour aider les Unions où la récolte de pommes de terre avait à nouveau été touchée), les dettes restantes furent consolidées. Elles représentaient une somme de plus de 3,7 millions de livres, que la pauvreté de l'Irlande ne lui permit pas d'acquitter, de telle sorte qu'en 1853, le gouvernement annonça l'annulation de cette dette, avec pour contrepartie la mise en place d'autres impôts. Thomas P. O'NEILL, " The Organisation and Administration of Relief, 1845-52 », in R. DUDLEY EDWARDS et T. Desmond WILLIAMS (eds.), The Great Famine : Studies in Irish History 1845-52 [1956], Dublin : The Lilliput Press, 1994, p. 254.

29. On peut ici supposer que, de surcroît, il pouvait être difficile de faire clairement la différence entre les symptômes d'une dénutrition extrême accompagnée d'une infection mineure et ceux de la fièvre typhoïde.

30. Voir Brian M. WALKER (ed.), Parliamentary Election Results in Ireland, 1801-1922, Dublin : Royal Irish Academy, pp. 75-79 ; et Christine KINEALY, Repeal and Revolution: 1848 in Ireland, Manchester : Manchester University Press, 2009.

\section{RÉSUMÉS}

La Loi sur les Pauvres irlandaise de 1838 n'avait pas pour finalité de parer à une famine à l'échelle de l'île tout entière. Ce fut toutefois ce dispositif que le gouvernement s'efforça d'imposer comme 
le cadre de la résolution du problème à partir de l'été 1846. Cet article propose de remonter aux fondements idéologiques de cette loi, afin de déterminer ce qu'elle était censée résoudre - sans y parvenir.

The Poor Law (Ireland) Act had never been intended to cater for such a disaster as the Famine. However, it was the apparatus through which the British government sought to solve the 'Irish crisis' from the summer of 1846 onwards. This paper means to challenge the implicit assumptions at work behind the law, in order to account for its failure in its self-prescribed duty.

\section{AUTEUR}

\section{ANNE-CATHERINE DE BOUVIER}

Université de Caen - Basse Normandie 